\title{
Convex solutions of the multi-valued iterative equation of order $n$
}

\section{Xiaobing Gong*}

\author{
"Correspondence: \\ xbgong@163.com \\ Department of Mathematics, \\ Sichuan University, Chengdu, \\ Sichuan 610064, P.R. China \\ College of Mathematics and \\ Information Science, Neijiang \\ Normal University, Neijiang, Sichuan \\ 641112, P.R. China
}

\begin{abstract}
A multi-valued iterative functional equation of order $n$ is considered. A result on the existence and uniqueness of $K$-convex solutions in some class of multifunctions is presented.
\end{abstract}

MSC: 39B12; 37E05; 54C60

Keywords: multifunction; functional equation; iteration; $K$-convex; upper semi-continuity

\section{Introduction}

As indicated in the books $[1,2]$ and the surveys [3, 4], the polynomial-like iterative equation

$$
\lambda_{1} f(x)+\lambda_{2} f^{2}(x)+\cdots+\lambda_{n} f^{n}(x)=F(x), \quad x \in S,
$$

where $S$ is a subset of a linear space over $\mathbb{R}, F: S \rightarrow S$ is a given function, $\lambda_{i} \mathrm{~s}(i=1, \ldots, n)$ are real constants, $f: S \rightarrow S$ is the unknown function, and $f^{i}$ is the $i$ th iterate of $f$, i.e., $f^{i}(x)=$ $f\left(f^{i-1}(x)\right)$ and $f^{0}(x)=x$ for all $x \in S$, is one of the important forms of a functional equation since the problem of iterative roots and the problem of invariant curves can be reduced to the kind of equations. Many works have been contributed to studying single-valued solutions for Eq. (1.1); for example, in [5-11] for the case of linear $F$, [12, 13] for $n=2$, [14] for general $n,[15,16]$ for smoothness, [17] for analyticity, [18-20] for convexity, [21-23] in high-dimensional spaces. However, a multifunction (called multi-valued function or setvalued map sometimes) is an important class of mappings often used in control theory [24], stochastics [25], artificial intelligence [26], and economics [27]. Hence, it gets more interesting to study multi-valued solutions for Eq. (1.1), i.e., the equation

$$
\lambda_{1} F(x)+\lambda_{2} F^{2}(x)+\cdots+\lambda_{n} F^{n}(x)=G(x), \quad x \in I:=[a, b]
$$

where $n \geq 2$ is an integer, $\lambda_{i} \mathrm{~s}(i=1, \ldots, n)$ are real constants, $G$ is a given multifunction, and $F$ is an unknown multifunction. Here the $i$ th iterate $F^{i}$ of the multifunction $F$ is defined recursively as

$$
F^{i}(x):=\bigcup\left\{F(y): y \in F^{i-1}(x)\right\}
$$

(c) 2012 Gong; licensee Springer. This is an Open Access article distributed under the terms of the Creative Commons Attribution License (http://creativecommons.org/licenses/by/2.0), which permits unrestricted use, distribution, and reproduction in any medium, provided the original work is properly cited. 
and $F^{0}(x): \equiv\{x\}$ for all $x \in I$. In 2004, Nikodem and Zhang [28] discussed Eq. (1.2) for $n=2$ with an increasing upper semi-continuous (USC) multifunction $G$ on $I=[a, b]$ and proved the existence and uniqueness of USC solutions under the assumption that $G$ has fixed points $a$ and $b$ and $\lambda_{1}, \lambda_{2}$ are both constants such that $\lambda_{1}>\lambda_{2} \geq 0$ and $\lambda_{1}+\lambda_{2}=1$. As pointed out in [29], the generalization to USC multifunctions for Eq. (1.1) is rather difficult even if $n=2$. Hence, discussing Eq. (1.2) for $n \geq 3$ evokes great interest, but the greatest difficulty is that the multifunction has no Lipschitz condition. In 2011, this difficulty was overcome by introducing the class of unblended multifunctions, the existence of USC multi-valued solutions for a modified form of the equation

$$
\lambda_{1} F(x)=G(x)-\lambda_{2} F^{2}(x)-\cdots-\lambda_{n} F^{n}(x), \quad x \in I,
$$

was proved in [29]. $K$-convex multifunctions, which are generalization of vector-valued convex functions, have wide applications in optimization ( $c f$. [30]) and play an important role in various questions of convex analysis ( $c f$. [31]). However, up to now, there are no results on convexity of multi-valued solutions for the iterative equation (1.2). In this note, we study the convexity of multi-valued solutions for Eq. (1.2). We prove the existence and uniqueness of $K$-convex solutions in some class of multifunctions for Eq. (1.3).

\section{$2 K$-convex multifunctions}

As in [30], let $X$ and $Y$ be linear spaces and $K \subset Y$ be a convex cone, i.e., $K+K \subset K$ and $\lambda K \subset K$ for all $\lambda \geq 0$. Let $\Omega \subset X$ be a convex set. A multifunction $T: X \rightarrow Y$ is said to be $K$-convex on $\Omega$ if

$$
\lambda T(x)+(1-\lambda) T(y) \subset T(\lambda x+(1-\lambda) y)+K, \quad \forall x, y \in \Omega, \lambda \in[0,1] .
$$

A convex multifunction [32] may be stated as $\theta$-convex and the convexity of a real-valued function may be stated as $\mathbb{R}^{+}$-convex, and concavity as $\mathbb{R}^{-}$-convex, where $\mathbb{R}^{+}:=[0,+\infty)$ and $\mathbb{R}^{-}:=(-\infty, 0]$. Let $\mathcal{F}(I)$ be the set of all multifunctions $F: I \rightarrow c c(I)$, where $c c(I)$ denotes the family of all nonempty closed subintervals of $I$.

Considering $\mathbb{R}^{+}$-convex multifunctions and $\mathbb{R}^{-}$-convex multifunctions, the following lemmas are obvious.

Lemma 2.1 Let $F(x) \in \mathcal{F}(I)$. Then the multifunction $F(x)$ is $\mathbb{R}^{+}$-convex on I if and only if

$$
\min \left(\lambda F\left(x_{1}\right)+(1-\lambda) F\left(x_{2}\right)\right) \geq \min F\left(\lambda x_{1}+(1-\lambda) x_{2}\right), \quad \forall x_{1}, x_{2} \in I, \lambda \in[0,1] .
$$

Lemma 2.2 Let $F(x) \in \mathcal{F}(I)$. Then the multifunction $F(x)$ is $\mathbb{R}^{-}$-convex on I if and only if

$$
\max \left(\lambda F\left(x_{1}\right)+(1-\lambda) F\left(x_{2}\right)\right) \leq \max F\left(\lambda x_{1}+(1-\lambda) x_{2}\right), \quad \forall x_{1}, x_{2} \in I, \lambda \in[0,1] .
$$

\section{Some lemmas}

In order to prove our main results, we give the following useful property $(c f .[33,34])$.

Lemma 3.1 For $A, B, C, D \in c c(I)$ and for an arbitrary real $\lambda$, the following properties hold:

(a) $h(A+C, B+C)=h(A, B)$, 
(b) $h(\lambda A, \lambda B)=|\lambda| h(A, B)$,

(c) $h(A+C, B+D) \leq h(A, B)+h(C, D)$,

where

$$
h(A, B)=\max \{\sup \{d(x, B): x \in A\}, \sup \{d(y, A): y \in B\}\} .
$$

As defined in [32, Definition 3.5.1], a multifunction $F: I \rightarrow c c(I)$ is increasing (resp. strictly increasing) if $\max F\left(x_{1}\right) \leq \min F\left(x_{2}\right)$ (resp. $\left.\max F\left(x_{1}\right)<\min F\left(x_{2}\right)\right)$ for all $x_{1}, x_{2} \in I$ with $x_{1}<x_{2}$. A multifunction $F: I \rightarrow c c(I)$ is upper semi-continuous (USC) at a point $x_{0} \in I$ if for every open set $v \subset \mathbb{R}$ with $F\left(x_{0}\right) \subset V$, there exists a neighborhood $U_{x_{0}}$ of $x_{0}$ such that $F(x) \subset V$ for every $x \in U_{x_{0}}$. $F$ is USC on $I$ if it is USC at every point in $I$. For convenience, let

$\operatorname{USIC}^{+}(I):=\left\{F \in \mathcal{F}(I): F\right.$ is USC, strictly increasing and $\mathbb{R}^{+}$-convex on $\left.I\right\}$

and

$\operatorname{USIC}^{-}(I):=\left\{F \in \mathcal{F}(I): F\right.$ is USC, strictly increasing and $\mathbb{R}^{-}$-convex on $\left.I\right\}$.

Remark 3.1 If $F \in \operatorname{USIC}^{+}(I)$ (resp. $\left.\operatorname{USIC}^{-}(I)\right), I=[a, b]$, then $F$ must be single-valued on $[a, b)$ (resp. $(a, b])$.

Lemma 3.2 $F_{1} \circ F_{2} \in \operatorname{USIC}^{+}(I)\left(r e s p . \operatorname{USIC}^{-}(I)\right)$ for $F_{1}, F_{2} \in \operatorname{USIC}^{+}(I)\left(r e s p . \operatorname{USIC}^{-}(I)\right)$.

Proof By Lemma 2.2 in [29], we only need to prove that $F_{1} \circ F_{2}$ is $\mathbb{R}^{+}$-convex on $I$ (resp. $\mathbb{R}^{-}$-convex on $I$ ). We first prove that $F_{1} \circ F_{2}$ is $\mathbb{R}^{+}$-convex on $I$ for $F_{1}, F_{2} \in \operatorname{USIC}^{+}(I)$. By Lemma 2.1, the fact that $F_{2}$ is $\mathbb{R}^{+}$-convex on $I$ implies that

$$
\min \left(\lambda F_{2}\left(x_{1}\right)+(1-\lambda) F_{2}\left(x_{2}\right)\right) \geq \min F_{2}\left(\lambda x_{1}+(1-\lambda) x_{2}\right), \quad \forall x_{1}, x_{2} \in I, \lambda \in[0,1] .
$$

Hence, for all $y \in \lambda F_{2}\left(x_{1}\right)+(1-\lambda) F_{2}\left(x_{2}\right)$,

$$
y \geq \min F_{2}\left(\lambda x_{1}+(1-\lambda) x_{2}\right)
$$

holds. Note that $F_{1}$ is strictly increasing. Consequently,

$$
\begin{aligned}
\min F_{1}(y) & \geq \min F_{1}\left(\min F_{2}\left(\lambda x_{1}+(1-\lambda) x_{2}\right)\right) \\
& =\min F_{1} \circ F_{2}\left(\lambda x_{1}+(1-\lambda) x_{2}\right) .
\end{aligned}
$$

So

$$
\begin{aligned}
\min F_{1}\left(\lambda F_{2}\left(x_{1}\right)+(1-\lambda) F_{2}\left(x_{2}\right)\right) & =\min \bigcup\left\{F_{1}(y): y \in \lambda F_{2}\left(x_{1}\right)+(1-\lambda) F_{2}\left(x_{2}\right)\right\} \\
& \geq \min F_{1} \circ F_{2}\left(\lambda x_{1}+(1-\lambda) x_{2}\right) .
\end{aligned}
$$

By

$$
\min \left(\lambda F_{1} \circ F_{2}\left(x_{1}\right)+(1-\lambda) F_{1} \circ F_{2}\left(x_{2}\right)\right)=\lambda \min F_{1} \circ F_{2}\left(x_{1}\right)+(1-\lambda) \min F_{1} \circ F_{2}\left(x_{2}\right),
$$


we have

$$
\begin{aligned}
\min \left(\lambda F_{1} \circ F_{2}\left(x_{1}\right)+(1-\lambda) F_{1} \circ F_{2}\left(x_{2}\right)\right) & \geq \min F_{1}\left(\lambda \min F_{2}\left(x_{1}\right)+(1-\lambda) \min F_{2}\left(x_{2}\right)\right) \\
& =\min F_{1}\left(\min \left(\lambda F_{2}\left(x_{1}\right)+(1-\lambda) F_{2}\left(x_{2}\right)\right)\right) \\
& =\min F_{1}\left(\lambda F_{2}\left(x_{1}\right)+(1-\lambda) F_{2}\left(x_{2}\right)\right)
\end{aligned}
$$

because $F_{1}$ is $\mathbb{R}^{+}$-convex. Hence, by (3.1)

$$
\begin{aligned}
& \min \left(\lambda F_{1} \circ F_{2}\left(x_{1}\right)+(1-\lambda) F_{1} \circ F_{2}\left(x_{2}\right)\right) \\
& \quad \geq \min F_{1} \circ F_{2}\left(\lambda x_{1}+(1-\lambda) x_{2}\right), \quad \forall x_{1}, x_{2} \in I, \lambda \in[0,1] .
\end{aligned}
$$

$F_{1} \circ F_{2} \in \mathrm{USIC}^{+}(I)$ is proved.

Next, we prove $F_{1} \circ F_{2}$ is $\mathbb{R}^{-}$-convex on $I$ for $F_{1}, F_{2} \in \operatorname{USIC}^{-}(I)$. By Lemma 2.2 , the fact that $F_{2}$ is $\mathbb{R}^{-}$-convex on $I$ implies that

$$
\max \left(\lambda F_{2}\left(x_{1}\right)+(1-\lambda) F_{2}\left(x_{2}\right)\right) \leq \max F_{2}\left(\lambda x_{1}+(1-\lambda) x_{2}\right), \quad \forall x_{1}, x_{2} \in I, \lambda \in[0,1] .
$$

Hence, for all $y \in \lambda F_{2}\left(x_{1}\right)+(1-\lambda) F_{2}\left(x_{2}\right)$,

$$
y \leq \max F_{2}\left(\lambda x_{1}+(1-\lambda) x_{2}\right)
$$

holds. Note that $F_{1}$ is strictly increasing. Consequently,

$$
\begin{aligned}
\max F_{1}(y) & \leq \max F_{1}\left(\max F_{2}\left(\lambda x_{1}+(1-\lambda) x_{2}\right)\right) \\
& =\max F_{1} \circ F_{2}\left(\lambda x_{1}+(1-\lambda) x_{2}\right) .
\end{aligned}
$$

So

$$
\begin{aligned}
\max F_{1}\left(\lambda F_{2}\left(x_{1}\right)+(1-\lambda) F_{2}\left(x_{2}\right)\right) & =\max \bigcup\left\{F_{1}(y): y \in \lambda F_{2}\left(x_{1}\right)+(1-\lambda) F_{2}\left(x_{2}\right)\right\} \\
& \leq \max F_{1} \circ F_{2}\left(\lambda x_{1}+(1-\lambda) x_{2}\right) .
\end{aligned}
$$

By

$$
\begin{aligned}
& \max \left(\lambda F_{1} \circ F_{2}\left(x_{1}\right)+(1-\lambda) F_{1} \circ F_{2}\left(x_{2}\right)\right) \\
& \quad=\lambda \max F_{1} \circ F_{2}\left(x_{1}\right)+(1-\lambda) \max F_{1} \circ F_{2}\left(x_{2}\right),
\end{aligned}
$$

it follows that

$$
\begin{aligned}
\max \left(\lambda F_{1} \circ F_{2}\left(x_{1}\right)+(1-\lambda) F_{1} \circ F_{2}\left(x_{2}\right)\right) & \leq \max F_{1}\left(\lambda \max F_{2}\left(x_{1}\right)+(1-\lambda) \max F_{2}\left(x_{2}\right)\right) \\
& =\max F_{1}\left(\max \left(\lambda F_{2}\left(x_{1}\right)+(1-\lambda) F_{2}\left(x_{2}\right)\right)\right) \\
& =\max F_{1}\left(\lambda F_{2}\left(x_{1}\right)+(1-\lambda) F_{2}\left(x_{2}\right)\right)
\end{aligned}
$$


because $F_{1}$ is $\mathbb{R}^{-}$-convex. Hence, by (3.2)

$$
\begin{aligned}
& \max \left(\lambda F_{1} \circ F_{2}\left(x_{1}\right)+(1-\lambda) F_{1} \circ F_{2}\left(x_{2}\right)\right) \\
& \quad \leq \max F_{1} \circ F_{2}\left(\lambda x_{1}+(1-\lambda) x_{2}\right), \quad \forall x_{1}, x_{2} \in I, \lambda \in[0,1] .
\end{aligned}
$$

This completes the proof of $F_{1} \circ F_{2} \in \operatorname{USIC}^{-}(I)$.

Define

$$
\begin{aligned}
\operatorname{USIC}^{+*}(I):= & \left\{F \in \operatorname{USIC}^{+}(I): \min F(x)>x, x \in \operatorname{int} I\right\}, \\
\operatorname{USIC}^{-*}(I):= & \left\{F \in \operatorname{USIC}^{-}(I): \min F(x)>x, x \in \operatorname{int} I\right\}, \\
\operatorname{USIC}_{*}^{+}(I):= & \left\{F \in \operatorname{USIC}^{+}(I): \min F(x)<x, x \in \operatorname{int} I\right\}, \\
\operatorname{USIC}_{*}^{-}(I):=\left\{F \in \mathrm{USIC}^{-}(I): \min F(x)<x, x \in \operatorname{int} I\right\}, & \\
\operatorname{USIC}^{+}(I, m, M):= & \left\{F \in \mathrm{USIC}^{+}(I): m\left(x_{2}-x_{1}\right) \leq F\left(x_{2}\right)-F\left(x_{1}\right) \leq M\left(x_{2}-x_{1}\right),\right. \\
& \left.x_{1}<x_{2}, x_{1}, x_{2} \in \operatorname{int} I, \max F(b)=b\right\}, \\
\operatorname{USIC}^{-}(I, m, M):= & \left\{F \in \mathrm{USIC}^{-}(I): m\left(x_{2}-x_{1}\right) \leq F\left(x_{2}\right)-F\left(x_{1}\right) \leq M\left(x_{2}-x_{1}\right),\right. \\
& \left.x_{1}<x_{2}, x_{1}, x_{2} \in \operatorname{int} I, \min F(a)=a\right\},
\end{aligned}
$$

where $I=[a, b]$ and $M>m>0$.

Remark 3.2 The condition $\max F(b)=b$ for $F \in \operatorname{USIC}^{+}(I, m, M)(\min F(a)=a$ for $F \in$ $\left.\mathrm{USIC}^{-}(I, m, M)\right)$ guarantees that the iterations $F^{n}, n=2,3, \ldots$, are also multifunctions.

Lemma 3.3 $\operatorname{USIC}^{+}(I, m, M)$ and $\operatorname{USIC}^{-}(I, m, M)$ are complete metric spaces equipped with the distance

$$
D\left(F_{1}, F_{2}\right):=\sup \left\{h\left(F_{1}(x), F_{2}(x)\right): x \in I\right\} .
$$

Proof By Lemma 3.1 in [29], we only need to prove that if $\left\{F_{n}\right\} \subset \operatorname{USIC}^{\sigma}(I, m, M)$ such that $\lim _{n \rightarrow \infty} F_{n}=F(x)$ in $\operatorname{USI}(I, m, M)$, i.e.,

$$
\lim _{n \rightarrow \infty} D\left(F_{n}, F\right)=0,
$$

then $F(x)$ is $\mathbb{R}^{\sigma}$-convex on $I$, where $\sigma=+$ or $\sigma=-$. We first prove the case of $\operatorname{USIC}^{+}(I$, $m, M)$. By (3.3), we have $\lim _{n \rightarrow \infty} h\left(F_{n}(x), F(x)\right)=0, \forall x \in I$. Hence,

$$
\lim _{n \rightarrow \infty} h\left(F_{n}\left(\lambda x_{1}+(1-\lambda) x_{2}\right), F\left(\lambda x_{1}+(1-\lambda) x_{2}\right)\right)=0, \quad \forall x_{1}, x_{2} \in I, \lambda \in[0,1] .
$$

Note that by Lemma 3.1,

$$
\lim _{n \rightarrow \infty} h\left(\lambda F_{n}\left(x_{1}\right), \lambda F\left(x_{1}\right)\right)=0, \quad \forall x_{1} \in I, \lambda \in[0,1]
$$

and

$$
\lim _{n \rightarrow \infty} h\left((1-\lambda) F_{n}\left(x_{2}\right),(1-\lambda) F\left(x_{2}\right)\right)=0, \quad \forall x_{2} \in I, \lambda \in[0,1] .
$$


Hence,

$$
\begin{aligned}
& \lim _{n \rightarrow \infty} h\left(\lambda F_{n}\left(x_{1}\right)+(1-\lambda) F_{n}\left(x_{2}\right), \lambda F\left(x_{1}\right)+(1-\lambda) F\left(x_{2}\right)\right)=0, \\
& \quad \forall x_{1}, x_{2} \in I, \lambda \in[0,1] .
\end{aligned}
$$

By (3.4) and (3.5), we have for every $\varepsilon>0$, there exists $n_{0} \in \mathbb{N}$ such that

$$
F_{n_{0}}\left(\lambda x_{1}+(1-\lambda) x_{2}\right) \subset F\left(\lambda x_{1}+(1-\lambda) x_{2}\right)+\left(-\frac{\varepsilon}{2}, \frac{\varepsilon}{2}\right)
$$

and

$$
\lambda F\left(x_{1}\right)+(1-\lambda) F\left(x_{2}\right) \subset \lambda F_{n_{0}}\left(x_{1}\right)+(1-\lambda) F_{n_{0}}\left(x_{2}\right)+\left(-\frac{\varepsilon}{2}, \frac{\varepsilon}{2}\right),
$$

$\forall x_{1}, x_{2} \in I, \lambda \in[0,1]$. Consequently,

$$
\begin{aligned}
\min \left(\lambda F\left(x_{1}\right)+(1-\lambda) F\left(x_{2}\right)\right) & \geq \min \left(\lambda F_{n_{0}}\left(x_{1}\right)+(1-\lambda) F_{n_{0}}\left(x_{2}\right)\right)-\frac{\varepsilon}{2} \\
& \geq \min F_{n_{0}}\left(\lambda x_{1}+(1-\lambda) x_{2}\right)-\frac{\varepsilon}{2} \\
& \geq \min F\left(\lambda x_{1}+(1-\lambda) x_{2}\right)-\varepsilon
\end{aligned}
$$

because $F_{n_{0}}(x)$ is $\mathbb{R}^{+}$-convex on $I$. Hence,

$$
\min \left(\lambda F\left(x_{1}\right)+(1-\lambda) F\left(x_{2}\right)\right) \geq \min F\left(\lambda x_{1}+(1-\lambda) x_{2}\right),
$$

which shows that $F(x)$ is $\mathbb{R}^{+}$-convex on $I$.

Next we prove the case of $\sigma=-$. By (3.6) and (3.7), we have for every $\varepsilon>0$,

$$
\begin{aligned}
\max \left(\lambda F\left(x_{1}\right)+(1-\lambda) F\left(x_{2}\right)\right) & \leq \max \left(\lambda F_{n_{0}}\left(x_{1}\right)+(1-\lambda) F_{n_{0}}\left(x_{2}\right)\right)+\frac{\varepsilon}{2} \\
& \leq \max F_{n_{0}}\left(\lambda x_{1}+(1-\lambda) x_{2}\right)+\frac{\varepsilon}{2} \\
& \leq \max F\left(\lambda x_{1}+(1-\lambda) x_{2}\right)+\varepsilon
\end{aligned}
$$

because $F_{n_{0}}(x)$ is $\mathbb{R}^{-}$-convex on $I$. Hence,

$$
\max \left(\lambda F\left(x_{1}\right)+(1-\lambda) F\left(x_{2}\right)\right) \leq \max F\left(\lambda x_{1}+(1-\lambda) x_{2}\right)
$$

which shows that $F(x)$ is $\mathbb{R}^{-}$-convex on $I$. The proof is completed.

Define

$$
\begin{aligned}
& \operatorname{USIC}^{+*}(I, m, M):=\operatorname{USIC}^{+*}(I) \cap \operatorname{USIC}^{+}(I, m, M), \\
& \operatorname{USIC}_{*}^{+}(I, m, M):=\operatorname{USIC}_{*}^{+}(I) \cap \operatorname{USIC}^{+}(I, m, M), \\
& \mathrm{USIC}^{-*}(I, m, M):=\operatorname{USIC}^{-*}(I) \cap \operatorname{USIC}^{-}(I, m, M), \\
& \operatorname{USIC}_{*}^{-}(I, m, M):=\operatorname{USIC}_{*}^{-}(I) \cap \operatorname{USIC}^{-}(I, m, M),
\end{aligned}
$$


$\mathrm{USIC}_{*}^{+}(I, m, M)$ is a closed subset of $\operatorname{USIC}^{+}(I, m, M)$. $\operatorname{USIC}^{-*}(I, m, M)$ is a closed subset of $\operatorname{USIC}^{-}(I, m, M)$.

By Lemma 3.2, one can prove the following result.

Lemma 3.4 $F^{i} \in \operatorname{USIC}_{*}^{+}\left(I, m^{i}, M^{i}\right)\left(r e s p\right.$. $\left.\operatorname{USIC}^{-*}\left(I, m^{i}, M^{i}\right)\right)$ if $F \in \operatorname{USIC}_{*}^{+}(I, m, M)$ (resp. $\left.\mathrm{USIC}^{-*}(I, m, M)\right)$.

Lemma 3.5 If $F_{1}, F_{2} \in \mathrm{USIC}_{*}^{+}(I, m, M)\left(\right.$ resp. $\left.\mathrm{USIC}^{-*}(I, m, M)\right)$, then

$$
D\left(F_{1}^{i}, F_{2}^{i}\right) \leq\left(\sum_{j=0}^{i-1} M^{j}\right) D\left(F_{1}, F_{2}\right) .
$$

The proof of Lemma 3.5 is similar to that of Lemma 3.3 in [29]. We omit it here.

\section{Convex solutions}

Theorem 4.1 Suppose that $\lambda_{1}>0, \lambda_{i} \leq 0(i=2, \ldots, n)$ and $\sum_{i=1}^{n} \lambda_{i}=1$ and $G \in \operatorname{USIC}^{-*}(I$, $\left.m_{0}, M_{0}\right)$ with $M_{0}>m_{0}>0$. Then for arbitrary constants $M>m>0$ satisfying

$$
m \leq \frac{m_{0}+\sum_{i=2}^{n}\left|\lambda_{i}\right| m^{i}}{\lambda_{1}}, \quad M \geq \frac{M_{0}+\sum_{i=2}^{n}\left|\lambda_{i}\right| M^{i}}{\lambda_{1}}
$$

Eq. (1.3) has a unique solution $F \in \mathrm{USIC}^{-*}(I, m, M)$ if

$$
d:=\frac{1}{\lambda_{1}} \sum_{i=2}^{n}\left|\lambda_{i}\right| \sum_{j=0}^{i-1} M^{j}<1 .
$$

Proof Define the mapping $L: \operatorname{USIC}^{-*}(I, m, M) \rightarrow \mathcal{F}(I)$ by

$$
L F(x)=\frac{1}{\lambda_{1}}\left(G(x)-\sum_{i=2}^{n} \lambda_{i} F^{i}(x)\right), \quad \forall x \in I .
$$

By Lemma 3.2, $F^{i}(x), i=2, \ldots, n$ are strictly increasing $\mathbb{R}^{-}$-convex on $I$ because $F(x)$ is strictly increasing $\mathbb{R}^{-}$-convex. Since $G(x)$ is $\mathbb{R}^{-}$-convex on $I$ and $\max (A+B)=\max A+$ $\max B$, we have

$$
\begin{aligned}
\max & \left(\lambda L F\left(x_{1}\right)+(1-\lambda) L\left(x_{2}\right)\right) \\
= & \frac{1}{\lambda_{1}}\left(\max \lambda G\left(x_{1}\right)-\sum_{i=2}^{n} \lambda_{i} \max \lambda F^{i}\left(x_{1}\right)\right) \\
& +\frac{1}{\lambda_{1}}\left(\max (1-\lambda) G\left(x_{2}\right)-\sum_{i=2}^{n} \lambda_{i} \max (1-\lambda) F^{i}\left(x_{2}\right)\right) \\
= & \frac{1}{\lambda_{1}}\left(\max \left(\lambda G\left(x_{1}\right)+(1-\lambda) G\left(x_{2}\right)\right)\right)-\frac{1}{\lambda_{1}}\left(\sum_{i=2}^{n} \lambda_{i} \max \left(\lambda F^{i}\left(x_{1}\right)+(1-\lambda) F^{i}\left(x_{2}\right)\right)\right) \\
\leq & \frac{1}{\lambda_{1}}\left(\max G\left(\lambda x_{1}+(1-\lambda) x_{2}\right)-\sum_{i=2}^{n} \lambda_{i} \max F^{i}\left(\lambda x_{1}+(1-\lambda) x_{2}\right)\right) \\
= & \max L F\left(\lambda x_{1}+(1-\lambda) x_{2}\right), \quad \forall x_{1}, x_{2} \in I, \lambda \in[0,1] .
\end{aligned}
$$


Hence, $L F(x)$ is $\mathbb{R}^{-}$-convex on $I$. Obviously, $L F(x)$ is strictly increasing and $L F(x)>x$ for $x \in$ int $I$. Similar to the proof of Theorem 4.1 in [29], by Lemma 3.4 and condition (4.1), $L F(x) \in$ $\operatorname{USIC}^{-*}(I, m, M)$. Thus, we have proved that $L F(x)$ is a self-mapping on $\operatorname{USIC}^{-*}(I, m, M)$. By Lemma 3.5 and condition (4.2), $L$ is a contraction map. By Lemma 3.3, $\operatorname{USIC}^{-*}(I, m, M)$ is a complete metric space. Using Banach's fixed point principle, $L$ has a unique fixed point $F$ in $\operatorname{USIC}^{-*}(I, m, M)$, i.e.,

$$
F(x)=\frac{1}{\lambda_{1}}\left(G(x)-\sum_{i=2}^{n} \lambda_{i} F^{i}(x)\right), \quad \forall x \in I .
$$

This completes the proof.

We note the fact that $A+B \supset C$ if the sets $A, B, C$ satisfy $A=C-B$. Hence, every solution $F$ of Eq. (1.3) satisfies

$$
\lambda_{1} F(x)+\lambda_{2} F^{2}(x)+\cdots+\lambda_{n} F^{n}(x) \supset G(x), \quad \forall x \in I .
$$

We have the following result.

Corollary 4.1 Under the same conditions as in Theorem 4.1, there exists a multifunction $F \in \mathrm{USIC}^{-*}(I, m, M)$ such that (4.4) holds.

For multifunctions in the other class $\mathrm{USIC}_{*}^{+}(I, m, M)$, we have a similar result to Theorem 4.1. It can be proved similarly.

Theorem 4.2 Suppose that $\lambda_{1}>0, \lambda_{i} \leq 0(i=2, \ldots, n)$ and $\sum_{i=1}^{n} \lambda_{i}=1$ and $G \in \operatorname{USIC}_{*}^{+}(I$, $\left.m_{0}, M_{0}\right)$ with $M_{0}>m_{0}>0$. Then for arbitrary constants $M>m>0$ satisfying (4.1), Eq. (1.3) has a unique solution $F \in \operatorname{USIC}_{*}^{+}(I, m, M)$ if condition (4.2) holds.

Corollary 4.2 Under the same conditions as in Theorem 4.2, there exists a multifunction $F \in \mathrm{USIC}_{*}^{+}(I, m, M)$ such that (4.4) holds.

Remark 4.1 Although the assumption $F \in \operatorname{USIC}^{-*}(I)$ (or $\operatorname{USIC}_{*}^{+}(I)$ ) implies that $F$ is single-valued on $[a, b)$ (or $(a, b])$, but Eq. (1.3) cannot be considered on the interval $[a, b)$ (or $(a, b])$ as a single-valued case and the point $b$ (or $a$ ) as a multi-valued case, respectively, because there is no meaning at the point $b$ (or $a$ ).

Remark 4.2 By Remark 3.1, there is no strictly increasing $\mathbb{R}^{+}$-convex multifunction in $\mathrm{USIC}^{+*}(I, m, M)$. The same applies to the case of $\operatorname{USIC}_{*}^{-}(I, m, M)$. Consequently, Eq. (1.3) has no solution in $\operatorname{USIC}^{+*}(I, m, M)$ (resp. $\left.\operatorname{USIC}_{*}^{-}(I, m, M)\right)$.

Remark 4.3 By Theorem 4.1 and Theorem 4.2, we actually only prove the existence and uniqueness of $K$-convex ( $K=\mathbb{R}^{+}$and $K=\mathbb{R}^{-}$, i.e., $K$ is not a nontrivial convex cone) multivalued solutions for Eq. (1.3). In fact, there is no convex multi-valued (i.e., $\{0\}$-convex multi-valued) solutions for Eq. (1.3) in the multifunction class $\operatorname{USI}(I)$. Since $F(x)$ is a convex multi-valued function on $I$ if and only if

$$
\begin{aligned}
& \min \lambda F(x)+\min (1-\lambda) F(y) \geq \min F(\lambda x+(1-\lambda) y) \quad \text { and } \\
& \max \lambda F(x)+\max (1-\lambda) F(y) \leq \max F(\lambda x+(1-\lambda) y), \quad \forall x, y \in I, \lambda \in[0,1] .
\end{aligned}
$$


Hence, if Eq. (1.3) has a convex multi-valued solution $F$ in USI $(I)$, then $F$ must be strictly increasing on $I$, which is contradictory to (4.5).

Remark 4.4 We point out that we actually only have proved a special class of $K$-convex solutions, i.e., strictly increasing $K$-convex solutions of Eq. (1.3). It is very difficult to discuss $K$-convex solutions of Eq. (1.3) which are not strictly increasing because the method in [29] cannot be used. Discussing non-strictly-increasing $K$-convex solutions of Eq. (1.3) will be the subject of our next work.

\section{Examples}

We give an example to illustrate the applications of Theorem 4.1. Consider the equation

$$
\frac{5}{4} F(x)=G(x)+\frac{1}{4} F^{3}(x), \quad x \in I:=[0,1]
$$

where $n=3, \lambda_{1}=\frac{5}{4}, \lambda_{2}=0, \lambda_{3}=-\frac{1}{4}$ and

$$
G(x)= \begin{cases}{\left[0, \frac{2}{3}\right],} & x=0, \\ \frac{\sqrt{5 x+4}}{3}, & x \in(0,1] .\end{cases}
$$

Clearly, $G \in \mathrm{USIC}^{-*}\left(I, m_{0}, M_{0}\right)$, where

$$
m_{0}=\frac{5}{18}, \quad M_{0}=\frac{5}{12} .
$$

Let $m=\frac{1}{5}$ and $M=1$. It is easy to check that both (4.1) and (4.2) hold. Thus, by Theorem 4.1, Eq. (5.1) has a unique solution $F \in \operatorname{USIC}^{-*}(I, m, M)$.

Remark 5.1 Example (5.1) cannot be solved by known single-valued results.

\section{Competing interests}

The author declares that he has no competing interests.

\section{Acknowledgements}

The author is most grateful to the Editor for the careful reading of the manuscript and anonymous referees for valuable suggestions that helped in significantly improving an earlier version of this paper. This work was supported by Key Project of Sichuan Provincial Department of Education (12ZA086) (China).

\section{Received: 5 March 2012 Accepted: 23 October 2012 Published: 5 November 2012}

\section{References}

1. Kuczma, M, Choczewski, B, Ger, R: Iterative Functional Equations. Encyclopedia Math. Appl., vol. 32. Cambridge University Press, Cambridge (1990)

2. Targonski, G: Topics in Iteration Theory. Vandenhoeck \& Ruprecht, Göttingen (1981)

3. Baron, K, Jarczyk, W: Recent results on functional equations in a single variable, perspectives and open problems. Aequ. Math. 61, 1-48 (2001)

4. Zhang, J, Yang, L, Zhang, W: Some advances on functional equations. Adv. Math. (China) 24, 385-405 (1995)

5. Dhombres, JG: Itération linéaire d'ordre deux. Publ. Math. (Debr.) 24, 177-187 (1977)

6. Jarczyk, W: On an equation of linear iteration. Aequ. Math. 51, 303-310 (1996)

7. Matkowski, J, Zhang, W: On linear dependence of iterates. J. Appl. Anal. 6, 149-157 (2000)

8. Mukherjea, A, Ratti, JS: On a functional equation involving iterates of a bijection on the unit interval. Nonlinear Anal. 7, 899-908 (1983)

9. Mukherjea, A, Ratti, JS: On a functional equation involving iterates of a bijection on the unit interval II. Nonlinear Anal. 31, 459-464 (1998)

10. Tabor, J, Tabor, J: On a linear iterative equation. Results Math. 27, 412-421 (1995) 
11. Yang, D, Zhang, W: Characteristic solutions of polynomial-like iterative equations. Aequ. Math. 67, 80-105 (2004)

12. Malenica, M: On the solutions of the functional equation $\phi(x)+\phi^{2}(x)=F(x)$. Mat. Vesn. 6, 301-305 (1982)

13. Zhao, L: A theorem concerning the existence and uniqueness of solutions of the functional equation $\lambda_{1} f(x)+\lambda_{2} f^{2}(x)=F(x)$. J. Univ. Sci. Tech. 32, 21-27 (1983) (in Chinese)

14. Zhang, W: Discussion on the iterated equation $\sum_{i=1}^{n} \lambda_{i} f^{i}(x)=F(x)$. Chin. Sci. Bull. 32, 1444-1451 (1987)

15. Mai, J, Liu, X: Existence, uniqueness and stability of $C^{m}$ solutions of iterative functional equations. Sci. China Ser. A 43, 897-913 (2000)

16. Zhang, W: Discussion on the differentiable solutions of the iterated equation $\sum_{i=1}^{n} \lambda_{j} f^{i}(x)=F(x)$. Nonlinear Anal. 15, 387-398 (1990)

17. Si, J: Existence of locally analytic solutions of the iterated equation $\sum_{i=1}^{n} \lambda_{j} f^{i}(x)=F(x)$. Acta Math. Sin. 37, 590-600 (1994) (in Chinese)

18. Trif, T: Convex solutions to polynomial-like iterative equations on open intervals. Aequ. Math. 79, 315-325 (2010)

19. Xu, B, Zhang, W: Decreasing solutions and convex solutions of the polynomial-like iterative equation. J. Math. Anal. Appl. 329, 483-497 (2007)

20. Zhang, W, Nikodem, K, Xu, B: Convex solutions of polynomial-like iterative equations. J. Math. Anal. Appl. 315, 29-40 (2006)

21. Kulczycki, M, Tabor, J: Iterative functional equations in the class of Lipschitz functions. Aequ. Math. 64, 24-33 (2002)

22. Tabor, J, Żoldak, M: Iterative equations in Banach spaces. J. Math. Anal. Appl. 299, 651-662 (2004)

23. Zhang, W: Solutions of equivariance for a polynomial-like iterative equation. Proc. R. Soc. Edinb. A 130, 1153-1163 (2000)

24. Johansson, KH, Rantzer, A, Aström, KJ: Fast switches in relay feedback systems. Automatica 35, $539-552$ (1999)

25. Choi, C, Nam, D: Interpolation for partly hidden diffusion processes. Stoch. Process. Appl. 113, 199-216 (2004)

26. Lawry, J: A framework for linguistic modelling. Artif. Intell. 155, 1-39 (2004)

27. Starr, RM: General Equilibrium Theory. Cambridge University Press, Cambridge (1997)

28. Nikodem, K, Zhang, W: On a multivalued iterative equation. Publ. Math. 64, 427-435 (2004)

29. Xu, B, Nikodem, K, Zhang, W: On a multivalued iterative equation of order $n$. J. Convex Anal. 18, $673-686$ (2011)

30. Borwein, J: Multivalued convexity and optimization: a unified approach to inequality and equality constraints. Math. Program. 13, 183-199 (1977)

31. Kuroiwa, D, Tanaka, T, Ha, TXD: On cone convexity of set-valued maps. Nonlinear Anal., Theory Methods Appl. 30 1487-1496 (1997)

32. Aubin, JP, Frankowska, H: Set-Valued Analysis. Birkhäuser, Boston (1990)

33. Radström, H: An embedding theorem for space of convex sets. Proc. Am. Math. Soc. 3, 165-169 (1952)

34. Smajdor, W: Local set-valued solutions of the Jensen and Pexider functional equations. Publ. Math. 43, 255-263 (1993)

doi:10.1186/1029-242X-2012-258

Cite this article as: Gong: Convex solutions of the multi-valued iterative equation of order $n$. Journal of Inequalities and Applications 2012 2012:258.

\section{Submit your manuscript to a SpringerOpen ${ }^{\circ}$ journal and benefit from:}

- Convenient online submission

- Rigorous peer review

- Immediate publication on acceptance

- Open access: articles freely available online

- High visibility within the field

- Retaining the copyright to your article 